\title{
High-resolution whole-heart contrast-enhanced coronary MRA in 5 minutes with self-navigation and $100 \%$ gating efficiency
}

Jianing Pang ${ }^{1,2^{*}}$, Qi Yang ${ }^{3}$, Kuncheng $\mathrm{Li}^{3}$, Yi He ${ }^{4}$, Zhanming Fan ${ }^{4}$, Bin Sun ${ }^{5}$, Fabio S Raman ${ }^{6}$, Mark A Ahlman ${ }^{6}$, David Bluemke ${ }^{6,7}, X_{i a o m i n g ~ B i}{ }^{8}$, Jing An ${ }^{9}$, Daniel S Berman ${ }^{1}$, Debiao Li $^{1}$

From 17th Annual SCMR Scientific Sessions

New Orleans, LA, USA. 16-19 January 2014

\section{Background}

Contrast-enhanced (CE) whole-heart coronary MRA is a promising technique for CAD detection [1]. However, the current imaging time is relatively long and variable, and the spatial resolution is limited. The aim of this work is to develop and evaluate a $3 \mathrm{D}$ projection reconstruction (3DPR) based CE coronary MRA technique that achieves $(1.0 \mathrm{~mm})^{3}$ spatial resolution and 5 -minute scan time, which has been previously validated on non-contrast coronary MRA [2]. Similar to the previous work, we compare the apparent SNR of two undersampling levels with 10,000 and 20,000 radial projections to explore the impact of undersampling on image quality in the context of $\mathrm{CE}$ imaging.

\section{Methods}

We employed a 3-bolus contrast injection scheme: the first two boluses are in the size of $0.05 \mathrm{mmol} / \mathrm{kg}$ at 4 $\mathrm{ml} / \mathrm{s}$ intended for the stress and rest perfusion scans, and the remaining $0.10 \mathrm{mmol} / \mathrm{kg}$ is injected right before the coronary MRA at the same rate. We used an ECGgated, fat-saturated, inversion-recovery prepared spoiled gradient-echo sequence with 3DPR k-space trajectory for free-breathing data acquisition with self-navigated motion correction and integrated non-Cartesian sensitivity encoding acceleration [2]. We performed healthy volunteer studies $(\mathrm{N}=10)$ to compare the image quality at two undersampling levels, 10,000 projections and 20,000 projections, which correspond to scans times of around 5 and 10 minutes, respectively. The comparisons are made on apparent signal-to-noise ratio (aSNR),

Biomedical Imaging Research Institute, Cedars-Sinai Medical Center, Los Angeles, California, USA

Full list of author information is available at the end of the article measured as the ratio between the average signal within a blood ROI and the standard deviation within a background ROI.

\section{Results}

As shown in Figure 1, the average scan times of the 10,000 and 20,000 projection images were $5.4 \pm 0.4$ and $10.8 \pm 0.8$ minutes, and the average aSNR values were $22.20 \pm 3.51$ and $19.61 \pm 2.81$, respectively. The 10,000 projection images show significantly higher aSNR values due to the higher contrast concentration on the earlier stage of the acquisition. Additionally, the employed nonCartesian acceleration suppressed the streaking artifacts, therefore maintained the aSNR of the undersampled image despite a $50 \%$ scan time reduction. Example images are shown in Figure 2.

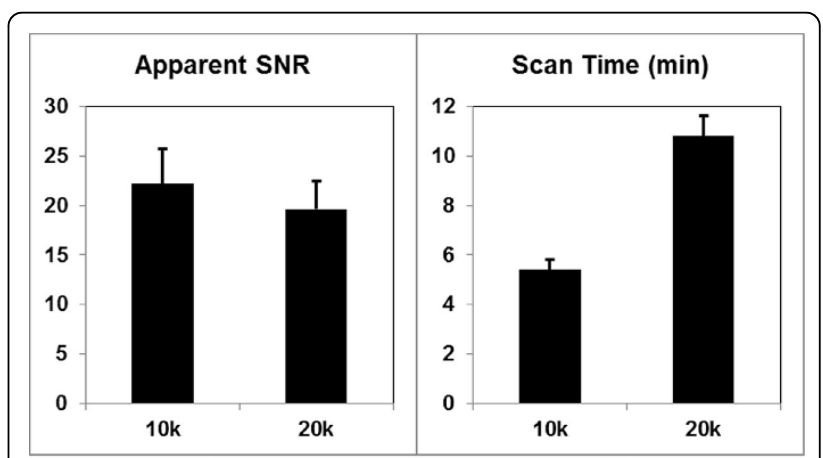

(a)

(b)

Figure 1 (a) the 10,000 projection images, acquired during the first 5 minutes of contrast injection, show significantly higher aSNR than the 20,000 projection images $(P<0.05)$; (b) it takes an average time of 5.4 minutes to acquire the 10,000 projection images, half of the time to acquire 20,000 projections. 


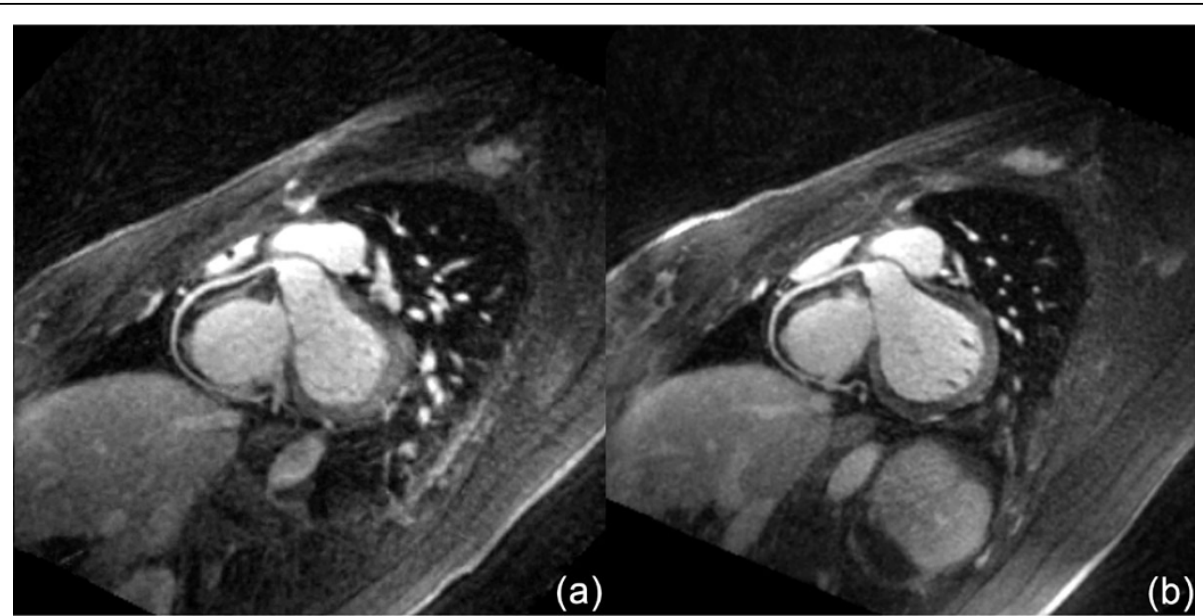

Figure 2 Example RCA visualizations. (a) 10,000 projections; (b) 20,000 projections

\section{Conclusions}

We have developed a CE coronary MRA technique that delivers good image quality at $(1.0 \mathrm{~mm})^{3}$ spatial resolution with scan time of 5 minutes. Further investigations are warranted on subjective image quality evaluation, protocol optimization, and CAD patient studies.

\section{Funding}

NIH Grant Numbers: HL38698, EB002623.

\section{Authors' details}

'Biomedical Imaging Research Institute, Cedars-Sinai Medical Center, Los Angeles, California, USA. ${ }^{2}$ Radiology and Biomedical Engineering,

Northwestern University, Chciago, Illinois, USA. ${ }^{3}$ Department of Radiology, Xuanwu Hospital of Capital Medical University, Beijing, China. ${ }^{4}$ Department of Radiology, Anzhen Hospital, Capital Medical University, Beijing, China. ${ }^{5}$ Fujian Medical University Union Hospital, Fuzhou City, China. ${ }^{6}$ Radiology and Imaging Sciences, National Institutes of Health, Bethesda, Maryland, USA. ${ }^{7}$ Molecular Biomedical Imaging Laboratory, National Institutes of Health, Bethesda, Maryland, USA. ${ }^{8}$ Siemens Medical Solutions, Los Angeles, California, USA. ${ }^{9}$ Siemens Medical Solutions, Beijing, China.

Published: 16 January 2014

\section{References}

1. Yang, et al: Circ Cardiovasc Imaging 2012.

2. Pang, et al: ISMRM 2013, 1295.

\section{doi:10.1186/1532-429X-16-S1-080}

Cite this article as: Pang et al: High-resolution whole-heart contrastenhanced coronary MRA in 5 minutes with self-navigation and 100\% gating efficiency. Journal of Cardiovascular Magnetic Resonance 201416 (Suppl 1):080.

\section{Submit your next manuscript to BioMed Central and take full advantage of:}

- Convenient online submission

- Thorough peer review

- No space constraints or color figure charges

- Immediate publication on acceptance

- Inclusion in PubMed, CAS, Scopus and Google Scholar

- Research which is freely available for redistribution 